\title{
Effects on clinical outcomes of intensifying triple oral antidiabetic drug (OAD) therapy by initiating insulin versus enhancing OAD therapy in patients with type 2 diabetes: A nationwide population-based, propensity-score-matched cohort study
}

\author{
Shihchen Kuo $\mathrm{PhD}^{1 *} \mid$ Chun-Ting Yang $\mathrm{MS}^{2 *} \mid$ Jin-Shang Wu MD ${ }^{3,4}$ Huang-Tz Ou PhD²,56
}

${ }^{1}$ Division of Metabolism, Endocrinology and Diabetes, Department of Internal Medicine, University of Michigan, Ann Arbor, Michigan

${ }^{2}$ Institute of Clinical Pharmacy and

Pharmaceutical Sciences, College of Medicine, National Cheng Kung University, Tainan,

Taiwan

${ }^{3}$ Department of Family Medicine, National Cheng Kung University Hospital, Tainan,

Taiwan

${ }^{4}$ Department of Family Medicine, College of Medicine, National Cheng Kung University,

Tainan, Taiwan

${ }^{5}$ Department of Pharmacy, College of Medicine, National Cheng Kung University, Tainan, Taiwan

${ }^{6}$ Department of Pharmacy, National Cheng Kung University Hospital, Tainan, Taiwan

Correspondence

Huang-Tz Ou, PhD, Institute of Clinical Pharmacy and Pharmaceutical Sciences, College of Medicine, National Cheng Kung University, 1 University Road, Tainan 7010, Taiwan.

Email: huangtz@mail.ncku.edu.tw

\section{Funding information}

This work was supported by the Ministry of Science and Technology, Taiwan, under grant (MOST 104-2320-B-006-008-MY3). The funder had no role in the design and conduct of the study; collection, management, analysis, and interpretation of the data; preparation, review, or approval of the manuscript; and decision to submit the manuscript for publication.

\begin{abstract}
Aims: To compare the effects of initiating insulin as a fourth-line antidiabetic therapy with the effects of enhancing oral antidiabetic drug (OAD) therapy in patients with type 2 diabetes mellitus (T2DM) with triple OAD therapy failure.

Materials and methods: We conducted a nationwide population-based, retrospective cohort study involving 1022 (without prevalent diabetes-related complications [PDRCs]) and 2077 (with/without PDRCs) propensity score-matched pairs of fourth-line insulin therapy users and enhanced OAD therapy users identified in the period 2004 to 2010. Clinical outcomes including a composite cardiovascular outcome (myocardial infarction, stroke, heart failure or ischaemic heart disease), peripheral vascular disease (PVD), hypoglycaemia and all-cause mortality were assessed up to 2013. Hypoglycaemia was adjusted in Cox models to consider its potential effect on study outcomes.

Results: In a T2DM cohort without PDRCs, fourth-line insulin therapy was not associated with greater risks of clinical outcomes, except hypoglycaemia (hazard ratio [HR] 1.45, 95\% confidence interval $[\mathrm{Cl}]$ 1.02-2.07), compared with enhanced OAD therapy. Among patients with T2DM with/without PDRCs, fourth-line insulin therapy was associated with greater risks of the composite cardiovascular outcome ( $\mathrm{HR} 1.23,95 \% \mathrm{Cl} 1.03-1.46)$, heart failure (HR $1.59,95 \% \mathrm{Cl}$ 1.12-2.25), ischaemic heart disease (HR 1.37, 95\% Cl 1.09-1.73), PVD (HR 1.17, 95\% Cl 1.00-1.36), hypoglycaemia (HR 1.49, 95\% Cl 1.20-1.85) and all-cause mortality (HR 1.48, 95\% $\mathrm{Cl}$ 1.01-2.17), but adjustment for hypoglycaemia significantly attenuated the risk of heart failure (HR 1.34, 95\% Cl 0.92-1.94), PVD (HR 1.15, 95\% Cl 0.98-1.34) and all-cause mortality (HR 1.30, 95\% Cl 0.84-1.99).

Conclusions: Initiation of fourth-line insulin therapy can be considered for patients with T2DM with triple OAD therapy failure, and the importance of awareness and prevention of hypoglycaemia among insulin-treated patients with T2DM cannot be overstated.
\end{abstract}

\section{KEYWORDS}

antidiabetic drug, cardiovascular disease, cohort study, hypoglycaemia, insulin therapy, type 2 diabetes

\section{1 | INTRODUCTION}

Early insulin initiation is suggested to preserve $\beta$-cell function, ${ }^{1-3}$ but in real-world clinical practice insulin is still commonly prescribed at a

*Shihchen Kuo and Chun-Ting Yang contributed equally to the work. later stage of an antidiabetic treatment course because of clinical inertia. ${ }^{4}$ Most patients with type 2 diabetes mellitus (T2DM) generally do not begin insulin therapy until they have experienced poor glycaemic control using three oral antidiabetic drugs (OADs). ${ }^{5}$ Insulin initiation is often delayed or patients have poor adherence to insulin therapy 
because of their fears about difficulty with injections, weight gain and hypoglycaemia. ${ }^{6-8}$ Clinicians may have concerns about the safety profile and unfavourable clinical outcomes of insulin, and thus they choose to either maximize doses of three OADs or add another OAD as enhanced $O A D$ therapy for patients with triple OAD therapy failure, regardless of the clear recommendation by the American Diabetes Association (ADA) ${ }^{9}$ to initiate insulin therapy for these patients.

Current evidence from randomized controlled trials (RCTs) and longitudinal cohort studies on the cardiovascular outcomes of insulin therapy in patients with T2DM appears controversial. Favourable cardiovascular outcomes of intensive glycaemic control using sulphonylureas or insulin versus conventional therapy using diet control were documented in the 10-year follow-up UK Prospective Diabetes Study ${ }^{10}$; however, other RCTs either showed no significant benefits of insulin therapy on cardiovascular outcomes ${ }^{11,12}$ or revealed a link between insulin-based therapy and a greater number of non-fatal cardiovascular events. ${ }^{13}$ Recently, two meta-analyses of RCTs suggested a neutral effect of insulin therapy on cardiovascular outcomes. ${ }^{14,15}$ These study findings should be interpreted with caution, however, because the studies included a limited number of cardiovascular events and shorter follow-up periods, the sulphonylureas used ${ }^{10,12}$ might also have had detrimental effects on cardiovascular disease (CVD), ${ }^{16}$ and the study populations were specific to either patients at an early stage of diabetes ${ }^{11}$ or those with existing CVD. ${ }^{12,13}$ Conversely, longitudinal cohort studies have shown an association between insulin therapy and increased risk of CVD and all-cause mortality in patients with T2DM, but the effects of insulin therapy were only assessed in patients at an early stage of the antidiabetic treatment course, with insulin being used as monotherapy, or as second-line or third-line antidiabetic treatment. ${ }^{17-24}$

Because T2DM is a progressive disease, most patients with T2DM eventually require insulin therapy. Although it is quite common in realworld clinical practice for insulin therapy to be initiated at a later stage of an antidiabetic treatment course, the effects of insulin used as a fourth-line antidiabetic treatment in patients with T2DM who have failed to achieve glycaemic control on triple OAD therapy remain unknown. The aim of the present study, therefore, was first to investigate the risk of clinical outcomes associated with intensifying triple OAD therapy by initiating insulin (ie, in fourth-line insulin users) versus those of enhancing OAD therapy (ie, in potential insulin use candidates [PICs]). Second, we sought to determine whether hypoglycaemia played a role in the relationship between insulin use and clinical outcomes. Hypoglycaemia has been shown to contribute to the risk of developing $\mathrm{CVD}^{25-27}$; thus, we hypothesized that the increased hypoglycaemia associated with insulin therapy might negatively impact the association between insulin therapy and clinical outcomes. Third, as basal insulin alone is recommended as the initial insulin regimen by the ADA, ${ }^{9}$ we performed subgroup analyses to investigate whether the effects of insulin therapy differed according to insulin regimen.

\section{2 | MATERIALS AND METHODS}

This study was conducted with permission from the Institutional Review Board of National Cheng Kung University Hospital (B-EX103-015).

\section{1 | Data source}

This was a retrospective cohort study using the Longitudinal Cohort of Diabetes Patients (LHDB) dataset for 1996 to 2013 from Taiwan's National Health Insurance Research Database (NHIRD) that was released by Taiwan's National Health Research Institutes. The NHIRD is population based and derived from claims data of the National Health Insurance (NHI) programme, a mandatory-enrolment, singlepayment system that covers $>99 \%$ of Taiwan's population. ${ }^{28}$ The LHDB is a national representative dataset for the population of patients with diabetes in Taiwan and has been validated by Taiwan's National Health Research Institutes for research purposes. ${ }^{29}$ This dataset consists of longitudinal data (eg, disease diagnosis and prescription utilization records) from a random sample of 120000 deidentified incident diabetes cases from each calendar year since 1999, which were tracked back to 1996 and followed up to 2013 .

\section{2 | Study population}

Study patients extracted from the LHDB were newly diagnosed with T2DM (International Classification of Diseases, ninth revision, Clinical Modification [ICD-9-CM] codes: 250.x0, 250.x2, x =0-9) for the period 1999 to 2010 . We excluded those aged $<18$ years, and those diagnosed with type 1 diabetes or gestational diabetes. The study cohort selection process is detailed in Figure S1.

\section{3 | Exposure to antidiabetic drugs}

The World Health Organization Anatomical Therapeutic Chemical Classification System was used to define antidiabetic drugs in the LHDB. The LHDB in 2004 to 2010 was used to identify the treatment exposure of interest. This period was chosen because long-acting insulin analogues (LAIAs) were reimbursed by the NHI programme from 2004, and ending the study period in 2010 allowed a follow-up period of at least 3 years (ie, 2011-2013). During 2004 to 2010, the index date for the fourth-line insulin group was defined as the first date of initiating fourth-line insulin prescription for triple OAD therapy regimens, and the index date for the PIC group was the first date of enhancing OAD therapy by either increasing three OADs up to maximal doses or adding a fourth-line OAD. Before the index date, all patients in the two study groups were on sub-maximal doses of at least one of three OADs. Type of insulin was confirmed by labels and drug licensing codes (Table S1). Maximal doses of OADs were defined according to information provided by drug product labels (Table S2).

\section{4 | Definition of clinical outcomes}

Primary outcomes included a composite outcome of non-fatal/fatal CVD (ie, myocardial infarction [MI], cerebrovascular disease, heart failure or ischaemic heart disease) and all-cause mortality. Secondary outcomes included individual CVD events, peripheral vascular disease (PVD) and hypoglycaemia. Using the ICD-9-CM codes, events of hospitalization for CVD and PVD were identified from the inpatient claims files, and events of hypoglycaemia were identified from the emergency department, inpatient and outpatient claims files (Table S3). The accuracy of disease diagnoses based on ICD-9-CM 
coding in the NHIRD has been validated in previous studies. ${ }^{30-35}$ For example, the positive predictive value for the diagnosis of myocardial infarction, ischaemic stroke and heart failure has been reported to be as high as $93 \%, 94 \%$ and $98 \%$, respectively. In addition, hypoglycaemic events were ascertained according to the validated definition (ie, having any of the following ICD-9-CM codes: 251.0, 251.1, 251.2, 250.3, 250.8 or 962.3 ). ${ }^{36,37}$ The operational definitions for confirming mortality status have also been validated and described in previous studies. $^{38,39}$

\section{5 | Statistical analyses}

Primary analyses of baseline complication-free patients were conducted on an intention-to-treat basis, where the follow-up for patients was from the index date until death, dropout or loss to follow-up from the $\mathrm{NHI}$ programme, occurrence of study outcomes, or the end of 2013, whichever came first. Considering confounding by indication and selection bias, one-to-one five-digit greedy propensityscore matching (PSM) ${ }^{40,41}$ was used to adjust for imbalanced patient characteristics between study groups, in which treatment status was a dependent variable and a comprehensive list of independent variables (Table 1, Table S4) ${ }^{37,42}$ was selected a priori based on clinical importance related to selection of antidiabetic drug regimens and study outcomes. The variables used for the PSM included demographics, comorbidities, diabetes-related complications, antidiabetic drugs and CVD-related medications. Standardized mean difference (SMD) was used to test the difference in patient characteristics between study groups, and an absolute value of SMD $>0.1$ indicated statistically significant imbalance of patient characteristics between study groups. $^{43-45}$

The incidence rate of study outcomes was calculated as the total number of events over the follow-up period divided by the number of person-years at risk. Cox proportional hazards models were used to compare risks of study outcomes between two propensity-scorematched cohorts. Hazard ratios (HRs) and two-tailed $95 \%$ confidence intervals (Cls) were computed. A two-tailed $P$ value of $<0.05$ was considered statistically significant. Cumulative sums of martingale-based residuals were used to check the proportional hazard assumption in Cox models. ${ }^{46}$ To consider the potential effect of hypoglycaemia on study outcomes, we adjusted for the presence of hypoglycaemia after the index date as a covariate in Cox models. Before adjustment for hypoglycaemia, we assessed the total effect of the fourth-line insulin therapy versus the enhanced OAD therapy on risks of CVDs and death, while after adjustment for hypoglycaemia, we assessed the remaining effect of the fourth-line insulin therapy after the partial effect of hypoglycaemia was considered. Akaike's information criterion (AIC) was used to evaluate the quality of model fit between models with and without adjustment for hypoglycaemia; a model with a lower AIC had a better fit.

Secondary analyses were conducted in a larger cohort incorporating the study cohort for primary analyses with those patients having prevalent diabetes-related complications (PDRCs) at baseline (1 year before index date). History of PRDCs was additionally considered in the PSM procedure. This analysis was conducted with the consideration that some patients with T2DM may have already had comorbid diseases before initiating insulin, and thus the secondary analysis results could be generalizable to the T2DM population in real-world clinical practice.

Sensitivity and subgroup analyses were performed using the study cohort for primary analyses. First, as-treated scenario analysis was conducted to account for over-estimation of the treatment effect from primary analyses, where non-adherence to medications was ignored. In addition to the censored definitions in primary analyses, patients were also censored when medication treatment patterns changed. Second, we redefined maximal doses of OADs according to clinicians' discretion/recommendations (Table S2) in selecting the PIC group to account for the real-world clinical practice variation in using maximal doses of OADs. Third, we refined the definitions for stable users of fourth-line insulin or enhanced OAD therapy by adding another criterion that fourth-line insulin or enhanced OAD therapy needed to last for at least 180 days after the index date. Fourth, compared with enhanced OAD therapy, subgroup analyses were conducted to evaluate the effects of different fourth-line insulin initiation regimens, including basal insulin (ie, intermediate-acting human insulin or LAIAs) alone and LAIAs alone. SAS software (version 9.4) was used for all analyses.

\section{3 | RESULTS}

\section{1 | Study cohort characteristics}

We identified 3959 complication-free patients, with 1186 in the fourth-line insulin group and 2773 in the PIC group (Figure S1). Table 1 shows patient characteristics by study group. After PSM, we included 1022 patients in each group for primary analyses; there was no statistical difference in patient characteristics between treatment groups. Table S5 shows the follow-up time for each study outcome in primary analyses.

\section{2 | Primary analyses}

The HR of fourth-line insulin users versus PICs was $1.37(95 \% \mathrm{Cl}$ $0.99-1.89)$ and 1.53 (95\% Cl 0.80-2.94) for the composite outcome of CVD and all-cause death, respectively (Table 2). The HR for individual CVD events ranged from 0.67 (95\% Cl 0.27-1.63) for $\mathrm{MI}$ to 1.64 (95\% $\mathrm{Cl} 0.77-3.46)$ for heart failure. The HR was 1.20 (95\% Cl 0.91-1.58) and 1.45 (95\% Cl 1.02-2.07) for PVD and hypoglycaemia, respectively. Adjustment for hypoglycaemia significantly reduced HRs for most study outcomes, eg, the HR decreased by $20 \%$, from 1.53 to 1.23 , for all-cause death. Hypoglycaemia-adjusted Cox models yielded lower AIC values for all study outcomes. Table S6 shows that the fourth-line insulin group had a higher proportion of experiencing hypoglycaemia.

\section{3 | Secondary analyses}

In secondary analyses, we identified 2077 patients with T2DM with or without PDRCs in each group after PSM; there was no statistical difference in patient characteristics between groups (Table S4). The HR of fourth-line insulin users versus PICs was significantly increased for the composite outcome of CVD (1.23, 95\% Cl 1.03-1.46), heart 
TABLE 1 Patient characteristics of fourth-line insulin users (fourth-line insulin) and potential insulin use candidates before and after propensity-score matching (primary analysis: Complication-free study cohort ${ }^{\mathrm{a}}$ )

\begin{tabular}{|c|c|c|c|c|c|c|}
\hline \multirow[b]{2}{*}{ Baseline characteristics } & \multicolumn{3}{|l|}{ Before PSM } & \multicolumn{3}{|l|}{ After PSM } \\
\hline & $\begin{array}{l}\text { Fourth-line insulin, } \\
n=1186\end{array}$ & $\begin{array}{l}\text { PICs, } \\
n=2773\end{array}$ & $S M D^{b}$ & $\begin{array}{l}\text { Fourth-line insulin, } \\
n=1022\end{array}$ & $\begin{array}{l}\text { PICs, } \\
n=1022\end{array}$ & $S M D^{b}$ \\
\hline Mean \pm SD age, years & $50.97 \pm 11.67$ & $53.22 \pm 10.73$ & -0.20 & $51.51 \pm 11.65$ & $51.46 \pm 10.42$ & 0.00 \\
\hline Women, \% & 39.04 & 41.90 & -0.06 & 39.24 & 39.14 & 0.00 \\
\hline \multicolumn{7}{|l|}{ Year of index date ${ }^{c}, \%$} \\
\hline 2004 & 4.38 & 6.35 & -0.09 & 4.79 & 5.48 & -0.03 \\
\hline 2006 & 9.27 & 10.31 & -0.03 & 10.37 & 9.00 & 0.05 \\
\hline 2007 & 18.47 & 11.86 & 0.18 & 15.26 & 15.56 & -0.01 \\
\hline 2008 & 19.22 & 14.57 & 0.12 & 17.81 & 18.49 & -0.02 \\
\hline 2009 & 23.02 & 25.17 & -0.05 & 24.56 & 23.87 & 0.02 \\
\hline 2010 & 18.97 & 26.61 & -0.18 & 20.94 & 21.62 & -0.02 \\
\hline Hypertension & 44.18 & 52.58 & -0.17 & 46.09 & 46.87 & -0.02 \\
\hline Dyslipidaemia & 60.03 & 60.19 & -0.00 & 60.67 & 60.18 & 0.01 \\
\hline Diabetic ketoacidosis & 1.69 & 0.40 & 0.13 & 0.98 & 1.08 & -0.01 \\
\hline $\begin{array}{l}\text { Hyperosmolar hyperglycaemic } \\
\text { state }\end{array}$ & 0.42 & 0.07 & 0.07 & 0.39 & 0.20 & 0.04 \\
\hline Hypoglycaemia & 1.01 & 0.69 & 0.04 & 1.08 & 1.17 & -0.01 \\
\hline Depression & 1.43 & 1.08 & 0.03 & 1.57 & 1.47 & 0.01 \\
\hline \multicolumn{7}{|l|}{ CIC category, \% } \\
\hline Cancer & 5.65 & 3.28 & 0.11 & 4.89 & 5.28 & -0.02 \\
\hline Gastrointestinal & 27.66 & 24.45 & 0.07 & 27.20 & 27.98 & -0.02 \\
\hline Metformin & 96.37 & 95.06 & 0.06 & 95.99 & 95.69 & 0.01 \\
\hline Sulphonylureas & 95.78 & 94.37 & 0.07 & 95.30 & 95.79 & -0.02 \\
\hline Meglitinides & 8.60 & 8.69 & -0.00 & 9.49 & 7.83 & 0.06 \\
\hline Thiazolidinediones & 64.67 & 49.22 & 0.32 & 61.15 & 60.18 & 0.02 \\
\hline a-glucosidase inhibitors & 38.79 & 54.17 & -0.31 & 42.47 & 41.59 & 0.02 \\
\hline DPP-4 inhibitors & 6.83 & 7.54 & -0.03 & 7.14 & 8.71 & -0.06 \\
\hline \multicolumn{7}{|l|}{ CVD-related medications } \\
\hline Lipid-modifying agents & 53.04 & 56.04 & -0.06 & 54.31 & 55.28 & -0.02 \\
\hline a-blockers & 2.19 & 2.38 & -0.01 & 2.45 & 2.05 & 0.03 \\
\hline$\beta$-blockers & 18.21 & 20.74 & -0.06 & 18.59 & 16.63 & 0.05 \\
\hline RAAS agents & 30.52 & 36.53 & -0.13 & 30.63 & 32.49 & -0.04 \\
\hline Diuretics & 11.97 & 13.16 & -0.04 & 12.13 & 10.47 & 0.05 \\
\hline Calcium channel blockers & 21.67 & 30.65 & -0.21 & 23.68 & 23.19 & 0.01 \\
\hline Class I and III antiarrhythmics & 0.34 & 0.43 & -0.02 & 0.29 & 0.49 & -0.03 \\
\hline Digoxin & 0.51 & 0.54 & -0.00 & 0.59 & 0.29 & 0.04 \\
\hline Vasodilators & 1.77 & 1.73 & 0.00 & 1.96 & 1.17 & 0.06 \\
\hline Antithrombotic agents & 17.88 & 18.68 & -0.02 & 18.40 & 16.73 & 0.04 \\
\hline $\begin{array}{l}\text { Mean } \pm \text { SD HbA1c tests in past } \\
1 \text { year }\end{array}$ & $3.26 \pm 1.80$ & $3.07 \pm 1.89$ & 0.11 & $3.23 \pm 1.83$ & $3.37 \pm 1.94$ & -0.07 \\
\hline
\end{tabular}


TABLE 1 (Continued)

\begin{tabular}{|c|c|c|c|c|c|c|}
\hline \multirow[b]{2}{*}{ Baseline characteristics } & \multicolumn{3}{|l|}{ Before PSM } & \multicolumn{3}{|l|}{ After PSM } \\
\hline & $\begin{array}{l}\text { Fourth-line insulin, } \\
n=1186\end{array}$ & $\begin{array}{l}\text { PICs, } \\
n=2773\end{array}$ & SMD $^{b}$ & $\begin{array}{l}\text { Fourth-line insulin, } \\
\mathrm{n}=1022\end{array}$ & $\begin{array}{l}\text { PICs, } \\
n=1022\end{array}$ & SMD ${ }^{b}$ \\
\hline \multicolumn{7}{|c|}{ Index agent ${ }^{\mathrm{e}}$ prescriber's specialty, $\%$} \\
\hline Family medicine & 13.07 & 24.41 & -0.29 & 15.07 & 15.66 & -0.02 \\
\hline Endocrinology & 58.35 & 29.10 & 0.62 & 52.15 & 50.78 & 0.03 \\
\hline Internal medicine & 17.71 & 24.77 & -0.17 & 20.35 & 22.21 & -0.05 \\
\hline Nephrology & 0.93 & 3.07 & -0.15 & 1.08 & 1.37 & -0.03 \\
\hline
\end{tabular}

Abbreviations: CIC, chronic illness with complexity; CVD, cardiovascular disease; DPP-4, dipeptidyl peptidase-4; HbA1c, glycated haemoglobin; OAD, oral antidiabetic drug; PIC, potential insulin use candidate; PSM, propensity-score matching; RAAS, renin-angiotensin-aldosterone system; SMD, standardized mean difference.

${ }^{a}$ Complication-free study cohort is defined as patients without diabetes-related complications (a) at 1 year before index date and (b) before stable use of fourth-line insulin in the fourth-line insulin users group and of enhanced OAD therapy in the PIC group.

${ }^{\mathrm{b}} \mathrm{SMD}>0.1$ or $\mathrm{SMD}<-0.1$ indicates significant difference in baseline characteristics between fourth-line insulin and PIC groups.

' Index date is defined as the first date of insulin prescribed for fourth-line insulin users or the first date of three OADs' maximal doses reached/the first date of fourth-line OAD added on for PICs.

d Diabetes duration was measured as the time from the first date of type 2 diabetes diagnosis to index date.

' Index agent is denoted as the first insulin prescription for fourth-line insulin users or the first prescription of three OADs' maximal doses reached/ fourth-line OAD added on for PICs.

TABLE 2 Incidence rates and hazard ratios of diabetes-related complications and death for fourth-line insulin users (fourth-line insulin) versus potential insulin use candidates after propensity-score matching (primary analysis: Complication-free study cohort ${ }^{\mathrm{a}}$ )

\begin{tabular}{|c|c|c|c|c|c|c|}
\hline \multirow[b]{2}{*}{ Complications } & \multicolumn{2}{|c|}{ Event/1000 person-years } & \multirow[b]{2}{*}{$\begin{array}{l}\text { Fourth-line insulin } \\
\text { vs PICs HR } \\
\text { (95\% CI) }\end{array}$} & \multirow[b]{2}{*}{$\begin{array}{l}\text { Hypoglycaemia-adjusted } \\
\text { HR }(95 \% \mathrm{Cl})\end{array}$} & \multicolumn{2}{|c|}{ Akaike information criterion (AIC) } \\
\hline & $\begin{array}{l}\text { Fourth-line } \\
\text { insulin } \\
(n=1022)\end{array}$ & $\begin{array}{l}\text { PICs } \\
(n=1022)\end{array}$ & & & $\begin{array}{l}\text { Without } \\
\text { adjustment for } \\
\text { hypoglycaemia }\end{array}$ & $\begin{array}{l}\text { With adjustment } \\
\text { for } \\
\text { hypoglycaemia }\end{array}$ \\
\hline MI & 2.05 & 3.31 & $0.67(0.27-1.63)$ & $0.60(0.22-1.65)$ & 28.92 & 25.17 \\
\hline $\begin{array}{l}\text { Cerebrovascular } \\
\text { diseases }\end{array}$ & 10.20 & 7.03 & $1.40(0.88-2.24)$ & $1.18(0.71-1.95)$ & 99.80 & 88.15 \\
\hline Heart failure & 4.67 & 2.94 & $1.64(0.77-3.46)$ & $1.66(0.74-3.70)$ & 40.50 & 38.67 \\
\hline $\begin{array}{l}\text { Ischaemic heart } \\
\text { diseases }\end{array}$ & 10.43 & 8.96 & $1.20(0.77-1.88)$ & $1.24(0.78-1.96)$ & 108.11 & 105.97 \\
\hline PVD & 26.52 & 23.98 & $1.20(0.91-1.58)$ & $1.16(0.87-1.53)$ & 277.64 & 274.03 \\
\hline
\end{tabular}

Abbreviations: CVD, cardiovascular disease; HR, hazard ratio; MI, myocardial infarction; NA, not applicable; PIC, potential insulin use candidate; PVD, peripheral vascular disease.

a Complication-free study cohort is defined as patients without diabetes-related complications (a) at 1 year before index date and (b) before stable use of fourth-line insulin in the fourth-line insulin users group and of enhanced OAD therapy in the PIC group.

${ }^{b}$ CVD composite included myocardial infarction, cerebrovascular disease, heart failure, ischaemic heart disease, arrhythmia, arteriosclerotic CVD, aortic aneurysm, cardiogenic shock and cardiac arrest.

failure $(1.59,95 \% \mathrm{Cl} 1.12-2.25)$, ischaemic heart disease $(1.37,95 \% \mathrm{Cl}$ 1.09-1.73), PVD (1.17, 95\% Cl 1.00-1.36), hypoglycaemia (1.49, 95\% $\mathrm{Cl} 1.20-1.85)$ and all-cause death (1.48, 95\% Cl 1.01-2.17). Likewise, hypoglycaemia adjustment significantly reduced the HRs and yielded lower AIC values for study outcomes. It is worth noting that the hypoglycaemia-adjusted HRs were no longer statistically significant for heart failure $(1.34,95 \% \mathrm{Cl} 0.92-1.94)$, PVD $(1.15,95 \% \mathrm{Cl}$ $0.98-1.34)$ and all-cause death (1.30, 95\% Cl 0.84-1.99 [Table S7]).

\subsection{Sensitivity and subgroup analyses}

Results of sensitivity analyses (Tables S8-S10) were consistent with those in primary analyses. In subgroup analyses, we selected two subgroups from the fourth-line insulin group, including: (a) 598 patients who initiated basal insulin alone vs. 598 propensity-scorematched PICs and (b) 517 patients who initiated LAIAs alone versus 517 propensity-score-matched PICs. The group of patients initiating LAIAs alone was a subgroup of patients initiating basal insulin alone. As shown in Table 3 and Tables S11 and S12, the HRs for each study outcome were not statistically significant, except for hypoglycaemia (1.45) in primary analyses, when comparing the entire fourth-line insulin group with the propensity-score-matched PIC group. Notably, however, there was an obvious decreased trend in HRs for the composite CVD outcome, $\mathrm{MI}+$ cerebrovascular disease and PVD for patients initiating any types of insulin, patients initiating basal insulin alone and patients initiating LAIAs alone compared with their 
TABLE 3 Hazard ratios of diabetes-related complications and death for fourth-line insulin users, fourth-line basal insulin users, fourth-line long-acting insulin analogue users vs. potential insulin use candidates after propensity-score matching (primary analysis: Complication-free study cohort $^{\mathrm{a}}$ )

\begin{tabular}{|c|c|c|c|}
\hline \multirow[b]{2}{*}{ Complications } & \multirow{2}{*}{$\begin{array}{l}\text { Primary analysis } \\
\text { Fourth-line insulin users vs. PICs HR } \\
(95 \% \mathrm{Cl})\end{array}$} & \multicolumn{2}{|l|}{ Subgroup analysis } \\
\hline & & $\begin{array}{l}\text { Fourth-line basal insulin users vs. PICs } \\
\text { HR }(95 \% \mathrm{CI})\end{array}$ & $\begin{array}{l}\text { Fourth-line LAIA users vs. PICs } \\
\text { HR }(95 \% \mathrm{Cl})\end{array}$ \\
\hline CVD composite ${ }^{\mathrm{b}}$ & $1.37(0.99-1.89)$ & $1.00(0.65-1.54)$ & $0.89(0.45-1.73)$ \\
\hline $\begin{array}{l}\text { MI + cerebrovascular } \\
\text { diseases }\end{array}$ & $1.22(0.81-1.86)$ & $0.76(0.42-1.38)$ & $0.65(0.23-1.87)$ \\
\hline $\begin{array}{l}\text { Peripheral vascular } \\
\text { diseases }\end{array}$ & $1.20(0.91-1.58)$ & $1.14(0.78-1.67)$ & $1.01(0.65-1.57)$ \\
\hline All-cause death & $1.53(0.80-2.94)$ & $1.37(0.55-3.42)$ & $-^{c}$ \\
\hline
\end{tabular}

Abbreviations: $\mathrm{Cl}$, confidence interval; CVD, cardiovascular disease; HR, hazard ratio; LAIA, long-acting insulin analogue; MI, myocardial infarction; OAD, oral antidiabetic drug; PIC, potential insulin use candidate.

${ }^{a}$ Complication-free study cohort is defined as patients without diabetes-related complications (a) at 1 year before index date and (b) before stable use of fourth-line insulin (or basal insulin alone or LAIAs alone) in the fourth-line insulin (or basal insulin or LAIA) users group and of enhanced OAD therapy in the PIC group. The group of patients initiating fourth-line basal insulin alone was a subgroup of patients initiating fourth-line insulin, and the group of patients initiating fourth-line LAIAs alone was a subgroup of patients initiating fourth-line basal insulin alone.

b CVD composite included myocardial infarction, cerebrovascular diseases, heart failure, ischaemic heart disease, arrhythmia, arteriosclerotic cardiovascular disease, aortic aneurysm, cardiogenic shock and cardiac arrest.

${ }^{\mathrm{c}} \mathrm{HR}$ could not be calculated because of the small number of events.

propensity-score-matched PIC group of patients; for example, the HR for the composite CVD outcome decreased from 1.37 to 1.00 and to 0.89 .

\section{4 | DISCUSSION}

The ADA explicitly recommends insulin therapy for patients with T2DM who have failed to achieve glycaemic control on triple therapy. ${ }^{9}$ In current clinical practice, however, a high proportion of such patients is still treated with either increasing doses of three OADs or by adding another OAD instead of initiating fourth-line insulin therapy. To our knowledge, this is the first large nationwide cohort study to evaluate the effects of initiating fourth-line insulin therapy in a real-world setting. First, we found that, in a T2DM cohort without PDRCs, initiating fourth-line insulin versus enhancing OAD therapy was not associated with increased risks of CVD, PVD or all-cause mortality. Second, in a T2DM population with or without PDRCs, fourth-line insulin was associated with increased risks of the composite outcome of CVD, heart failure, ischaemic heart disease, PVD and all-cause mortality. Third, fourth-line insulin was associated with a higher risk of hypoglycaemia than enhanced OAD therapy. Adjusting for hypoglycaemia reduced the risks of study outcomes and yielded lower AIC values of the models, suggesting that hypoglycaemia had a significant effect on the association between insulin therapy and increased CVD and all-cause mortality risks. Notably, among the T2DM population with or without PDRCs, adjusting for hypoglycaemia would neutralize excess risks of heart failure, PVD and all-cause mortality, which emphasizes the importance of awareness and prevention of hypoglycaemia among insulin-treated patients with T2DM. Fourth, compared with the propensity-score-matched PIC group, initiation of fourth-line insulin therapy using LAIAs alone was associated with a lower risk of the composite CVD outcome, $\mathrm{MI}+$ cerebrovascular disease and PVD.

\subsection{Effects of the fourth-line insulin versus enhanced OAD therapy on clinical outcomes}

The association of insulin therapy with incident CVD and mortality has been investigated previously, but evidence is lacking on the effects of initiating insulin as the fourth-line antidiabetic treatment in patients with T2DM. Previous longitudinal studies evaluated the effects of insulin when it was used as monotherapy, ${ }^{17,24}$ or secondline ${ }^{17-21,24}$ or third-line $e^{22-24}$ treatment, and revealed a harmful effect of insulin therapy on CVD and all-cause mortality. Unlike previous studies, we found that intensification of triple OAD therapy by initiating fourth-line insulin versus enhancing $O A D$ therapy was not associated with increased risks of the composite or individual outcomes of CVD, PVD or all-cause mortality in the complicationfree patients with T2DM, although it was associated with increased risks of some clinical outcomes among patients with or without PDRCs. Notably, one major concern in the previous studies is the bias attributable to confounding by indication because those studies compared insulin therapy with non-treatment, ${ }^{24}$ or metformin ${ }^{17,24}$ or sulphonylureas ${ }^{17}$ as monotherapy, or metformin + sulphonylureas $^{18,20,21}$ or metformin + dipeptidyl peptidase-4 (DPP-4) inibitors ${ }^{19}$ as dual therapy. Indeed, characteristics of insulin users are typically different from patients without any treatment ${ }^{24}$ or those with only one or two OADs. ${ }^{17-19,24}$ Unlike previous studies, we carefully identified a comparable group to the insulin-treated group and focused on evaluating the effects of fourth-line insulin therapy in patients with T2DM who failed to achieve glycaemic control on triple OAD therapy; in other words, all of our study patients had been candidates for insulin therapy. Moreover, we applied rigorous PSM approaches and identified baseline complication-free patients for primary analyses, which led to more comparable groups, minimized confounding by indication, and ensured causal inference in our study. 


\subsection{Role of hypoglycaemia in relationship between insulin therapy and clinical outcomes}

Hypoglycaemia is a major undesired effect of insulin therapy, and may play a role in a causal pathway between insulin therapy and risks of CVD and mortality. There is supporting evidence linking hypoglycaemia with increased risks of CVD and mortality in patients with T2DM or prediabetes, ${ }^{47-51}$ but one study reported no significant relationship between severe/symptomatic hypoglycaemic events and CVD-specific/ all-cause mortality in patients with T2DM starting insulin therapy. ${ }^{52}$ In the present study we found that the increased risks of clinical outcomes associated with insulin therapy became lower or even vanished after hypoglycaemia was adjusted for in the analyses, which provides the supporting evidence for the potential effect of hypoglycaemia on risks of CVD and mortality, and indicates the use of insulin per se may not be associated with increased risks of CVD and mortality (Table 2, Table S7). Considering all possible efforts will be made to minimize or avoid hypoglycaemia, such findings should reassure health professionals who may be reluctant to start insulin therapy because of the perception that it will have negative impacts on risks of CVD and mortality.

\subsection{Effects of initiating different types of insulin versus non-insulin therapy on clinical outcomes}

Basal insulins, especially LAIAs, lead to a lower risk of hypoglycaemia than other types of insulin. ${ }^{53,54}$ Considering that hypoglycaemia may contribute to CVD risk, it would be worth further assessing the effects of LAIAs versus other types of insulin or non-insulin therapy in T2DM; however, there are limited data on this topic. The ORIGIN trial $^{11}$ showed that glargine compared with non-insulin therapy had a neutral effect on cardiovascular outcomes. Another study using Swedish national registries ${ }^{19}$ demonstrated that second-line treatment using LAIAs versus DPP-4 inhibitors had a neutral effect on fatal/non-fatal CVDs and all-cause death, and that, compared with individual propensity-score-matched DPP-4 inhibitor users, users of LAIAs experienced a neutral effect on fatal/non-fatal CVD and allcause death, but users of short-acting insulin, pre-mixed insulin and intermediate-acting human insulin had increased risks. Our subgroup analyses added supporting evidence to the literature; these results might justify the suggestion of first considering LAIAs alone as the initial insulin therapy regimen for patients with T2DM.

\subsection{Strengths and limitations}

The present study has several strengths. First, it was based on a national representative cohort of patients with T2DM, with a longterm follow-up, and results could be applicable to patients who initiated insulin as a fourth-line antidiabetic treatment, which is a common prescription pattern of insulin use in real-world clinical practice, ${ }^{4}$ is a highly recommended treatment strategy by the ADA for patients with triple OAD therapy failure, ${ }^{9}$ and is suggested by Taiwan's NHI programme (because insulin is generally more expensive than OADs). Second, rigorous statistical approaches were used to minimize common potential biases, including confounding by indication, selection bias and immortal-time bias, to ensure causal inference. Third, a series of sensitivity and subgroup analyses were conducted to ensure the robustness of study findings. Particularly, we further broke down different types of insulin to analyse the effects of using LAIAs, which may fill a research gap in previous studies.

Several limitations should also be acknowledged. First, we used PSM to control for patient characteristics between study groups, but, like any study using administrative claims data, the residual effects attributable to unmeasured confounders could not be avoided. Selection bias may occur when the choice of initiating fourth-line insulin or enhancing OAD therapy is made through decisions based on physicianpatient discussions. Moreover, data on indicators of diabetes management, such as glycated haemoglobin, blood pressure or lipids, were unavailable in Taiwan's NHIRD. However, the duration and severity of T2DM, the use of OAD regimens and CVD-related medications, and the status of medical comorbidities were similar between study groups at baseline in our cohort study. Although a prospective RCT can overcome the potential confounding by measured or unmeasured covariates, there may be less motivation for initiation of an RCT to evaluate the fourthline antidiabetic treatment options. Second, we targeted insulin use as a fourth-line antidiabetic treatment, which would make our results unsuitable for explaining the effects of insulin initiation as monotherapy, second-line or third-line treatment. Third, the generalizability of our study findings may be limited to countries with universal health insurance. Lastly, the glucagon-like peptide-1 receptor agonist liraglutide and the sodium-glucose co-transporter-2 inhibitor empagliflozin were not available in Taiwan's NHI programme until 1 October 2012 and 1 May 2016, respectively, and thus our analyses did not possess sufficient power to assess the effects of these drugs on study outcomes because very few patients $(<0.01 \%)$ were prescribed these drugs.

In summary, our findings have important therapeutic implications, supporting the current clinical recommendations to initiate insulin therapy for patients with T2DM with triple OAD therapy failure and to provide comprehensive education on avoiding and treating hypoglycaemia in any insulin-treated patients. Future prospective trials are warranted to confirm our findings, especially the potential benefits of using LAIAs in T2DM on CVD and mortality.

\section{ACKNOWLEDGMENTS}

The project described was supported by Grant Number P30DK092926 (MCDTR and MCDTR's Methods and Measurement Core) from the National Institute of Diabetes and Digestive and Kidney Diseases. The funder had no role in the design and conduct of the study; collection, management, analysis, and interpretation of the data; preparation, review, or approval of the manuscript; and decision to submit the manuscript for publication.

\section{CONFLICT OF INTEREST}

None declared.

\section{Author contributions}

The author contributions to the study were as follows: conception and design, analysis and interpretation of the data, critical revision of 
the article for important intellectual content, and final approval of the article: S.K., C.-T.Y., J.-S.W. and H.T.O; drafting of the article and statistical expertise: S.K., C.-T.Y. and H.-T.O.; provision of study materials and patients, and administrative, technical and logistic support: H.-T. O.; collection and assembly of data: C.-T.Y. and H.-T.O.

\section{ORCID}

\section{Huang-Tz Ou (D) https://orcid.org/0000-0002-5475-7848}

\section{REFERENCES}

1. Lingvay I, Kaloyanova PF, Adams-Huet B, Salinas K, Raskin P. Insulin as initial therapy in type 2 diabetes. J Invest Med. 2007;55(2):62-68.

2. Harrison LB, Adams-Huet B, Raskin P, Lingvay I. $\beta$-cell function preservation after 3.5 years of intensive diabetes therapy. Diabetes Care. 2012;35(7):1406-1412.

3. Weng J, Li Y, Xu W, et al. Effect of intensive insulin therapy on $\beta$-cell function and glycaemic control in patients with newly diagnosed type 2 diabetes: a multicentre randomised parallel-group trial. Lancet. 2008; 371(9626):1753-1760.

4. Khunti K, Wolden ML, Thorsted BL, Andersen M, Davies MJ. Clinical inertia in people with type 2 diabetes. Diabetes Care. 2013;36(11): 3411-3417.

5. Lin SD, Tsai ST, Tu ST, et al. Glycosylated hemoglobin level and number of oral antidiabetic drugs predict whether or not glycemic target is achieved in insulin-requiring type 2 diabetes. Prim Care Diabetes. 2015;9(2):135-141.

6. Peyrot M, Rubin RR, Khunti K. Addressing barriers to initiation of insulin in patients with type 2 diabetes. Prim Care Diabetes. 2010;4: S11-S18.

7. Karter AJ, Subramanian U, Saha C, et al. Barriers to insulin initiation: the translating research into action for diabetes insulin starts project. Diabetes Care. 2010;33(4):733-735.

8. Chen $\mathrm{KW}$, Tseng $\mathrm{H}-\mathrm{M}$. The barriers to initiating insulin therapy among people with type 2 diabetes in Taiwan - A qualitative study. J Diabetes Metab. 2012;3:194.

9. American Diabetes Association. 8. Pharmacologic approaches to glycemic treatment. Diabetes Care. 2017;40(suppl 1):S64-S74.

10. Holman RR, Paul SK, Bethel MA, Matthews DR, Neil HA. 10-Year follow-up of intensive glucose control in type 2 diabetes. $N$ Engl $J$ Med. 2008;359:1577-1589.

11. Origin Trial Investigators. Basal insulin and cardiovascular and other outcomes in dysglycemia. N Engl J Med. 2012;2012(367):319-328.

12. Chaitman BR, Hardison RM, Adler D, et al. The bypass angioplasty revascularization investigation 2 diabetes randomized trial of different treatment strategies in type 2 diabetes mellitus with stable ischemic heart disease. Circulation. 2009;120(25):2529-2540.

13. Mellbin LG, Malmberg K, Norhammar A, Wedel H, Ryden L, DIGAMI 2 Investigators. Prognostic implications of glucose-lowering treatment in patients with acute myocardial infarction and diabetes: experiences from an extended follow-up of the diabetes mellitus insulin-glucose infusion in acute myocardial infarction (DIGAMI) 2 study. Diabetologia. 2011;54(6):1308-1317.

14. Anyanwagu U, Mamza J, Donnelly R, Idris I. Comparison of cardiovascular and metabolic outcomes in people with type 2 diabetes on insulin versus non-insulin glucose-lowering therapies (GLTs): a systematic review and meta-analysis of clinical trials. Diabetes Res Clin Pract. 2016;121:69-85.

15. Palmer SC, Mavridis D, Nicolucci A, et al. Comparison of clinical outcomes and adverse events associated with glucose-lowering drugs in patients with type 2 diabetes: a meta-analysis. JAMA. 2016;316(3): 313-324.

16. Monami M, Genovese S, Mannucci E. Cardiovascular safety of sulfonylureas: a meta-analysis of randomized clinical trials. Diabetes Obes Metab. 2013;15(10):938-953.

17. Currie CJ, Poole CD, Evans M, Peters JR, Morgan CL. Mortality and other important diabetes-related outcomes with insulin vs other antihyperglycemic therapies in type 2 diabetes. J Clin Endocrinol Metab. 2013;98(2):668-677.

18. Roumie CL, Greevy RA, Grijalva CG, et al. Association between intensification of metformin treatment with insulin vs sulfonylureas and cardiovascular events and all-cause mortality among patients with diabetes. JAMA. 2014;311(22):2288-2296.

19. Nystrom T, Bodegard J, Nathanson D, Thuresson M, Norhammar A, Eriksson JW. Second line initiation of insulin compared with DPP-4 inhibitors after metformin monotherapy is associated with increased risk of all-cause mortality, cardiovascular events, and severe hypoglycemia. Diabetes Res Clin Pract. 2017;123:199-208.

20. Ekstrom N, Svensson AM, Miftaraj M, et al. Cardiovascular safety of glucose-lowering agents as add-on medication to metformin treatment in type 2 diabetes: report from the Swedish National Diabetes Register. Diabetes Obes Metab. 2016;18(10):990-998.

21. Gamble JM, Thomas JM, Twells LK, Midodzi WK, Majumdar SR. Comparative effectiveness of incretin-based therapies and the risk of death and cardiovascular events in 38,233 metformin monotherapy users. Medicine (Baltimore). 2016;95(26):e3995.

22. Anyanwagu U, Mamza J, Mehta R, Donnelly R, Idris I. Cardiovascular events and all-cause mortality with insulin versus glucagon-like peptide-1 analogue in type 2 diabetes. Heart. 2016;102(19): 1581-1587.

23. Jil M, Rajnikant M, Richard D, Iskandar I. The effects of dual-therapy intensification with insulin or dipeptidylpeptidase-4 inhibitor on cardiovascular events and all-cause mortality in patients with type 2 diabetes: a retrospective cohort study. Diab Vasc Dis Res. 2017;14(4): 295-303.

24. Hippisley-Cox J, Coupland C. Diabetes treatments and risk of heart failure, cardiovascular disease, and all cause mortality: cohort study in primary care. BMJ. 2016;354:i3477.

25. Goto A, Arah OA, Goto M, Terauchi Y, Noda M. Severe hypoglycaemia and cardiovascular disease: systematic review and meta-analysis with bias analysis. BMJ. 2013;347:f4533.

26. Cha SA, Yun JS, Lim TS, et al. Severe hypoglycemia and cardiovascular or all-cause mortality in patients with type 2 diabetes. Diabetes Metab J. 2016;40(3):202-210.

27. Zoungas S, Patel A, Chalmers J, et al. Severe hypoglycemia and risks of vascular events and death. N Engl J Med. 2010;363:1410-1418.

28. Cheng T-M. Taiwan's new national health insurance program: genesis and experience so far. Health Aff. 2003;22(3):61-76.

29. Lin CC, Lai MS, Syu CY, Chang SC, Tseng FY. Accuracy of diabetes diagnosis in health insurance claims data in Taiwan. J Formos Med Assoc. 2005;104(3):157-163.

30. Cheng CL, Kao YH, Lin SJ, et al. Validation of the National Health Insurance Research Database with ischemic stroke cases in Taiwan. Pharmacoepidemiol Drug Saf. 2011;20:236-242.

31. Cheng CL, Lee CH, Chen PS, Li YH, Lin SJ, Yang YHK. Validation of acute myocardial infarction cases in the National Health Insurance Research database in Taiwan. J Epidemiol. 2014;24:500-507.

32. Chang CH, Lee YC, Tsai CT, et al. Continuation of statin therapy and a decreased risk of atrial fibrillation/flutter in patients with and without chronic kidney disease. Atherosclerosis. 2014;232(1):224-230.

33. Lin YS, Chen TH, Chi CC, et al. Different implications of heart failure, ischemic stroke, and mortality between nonvalvular atrial fibrillation and atrial flutter: a view from a national cohort study. J Am Heart Assoc. 2017;6(7):e006406.

34. Wu CS, Lai MS, Gau SS, Wang SC, Tsai HJ. Concordance between patient self-reports and claims data on clinical diagnoses, medication use, and health system utilization in Taiwan. PLoS One. 2014;9(12): e112257.

35. Sung SF, Hsieh CY, Lin HJ, Chen YW, Yang YH, Li CY. Validation of algorithms to identify stroke risk factors in patients with acute ischemic stroke, transient ischemic attack, or intracerebral hemorrhage in an administrative claims database. Int J Cardiol. 2016;215:277-282.

36. Ginde AA, Blanc PG, Lieberman RM, Camargo CA Jr. Validation of ICD-9-CM coding algorithm for improved identification of hypoglycemia visits. BMC Endocr Disord. 2008;8:4.

37. Chang HY, Weiner J, Richards TM, Bleich SN, Segal JB. Validating the adapted Diabetes Complications Severity Index in claims data. Am J Manag Care. 2012;18(11):721-726. 
38. Cheng C-L, Chien H-C, Lee C-H, Lin S-J, Yang Y-HK. Validity of in-hospital mortality data among patients with acute myocardial infarction or stroke in National Health Insurance Research Database in Taiwan. Int J Cardiol. 2015;201:96-101.

39. Ou HT, Chang KC, Li CY, Wu JS. Comparative cardiovascular risks of dipeptidyl peptidase 4 inhibitors with other second-and third-line antidiabetic drugs in patients with type 2 diabetes. $\mathrm{Br} \mathrm{J}$ Clin Pharmacol. 2017;83(7):1556-1570.

40. Dehejia RH, Wahba S. Propensity score-matching methods for nonexperimental causal studies. Rev Econ Stat. 2002;84(1):151-161.

41. Parsons LS. Reducing bias in a propensity score matched-pair sample using greedy matching techniques. Proceedings of the 26th Annual SAS Users Group International Conference. Cary, NC: SAS Institute Inc.; 2001:214-226.

42. Meduru P, Helmer D, Rajan M, Tseng C-L, Pogach L, Sambamoorthi U. Chronic illness with complexity: implications for performance measurement of optimal glycemic control. J Gen Intern Med. 2007;22(3): 408-418.

43. Cohen J. Statistical Power Analysis for the Behavioral Sciences. 2nd ed. Hillsdale, NJ: Lawrence Erlbaum Associates Publishers; 1988.

44. Austin PC. Using the standardized difference to compare the prevalence of a binary variable between two groups in observational research. Commun Stat Simul Comput. 2009;38(6):1228-1234.

45. Yang D, Dalton JE. A unified approach to measuring the effect size between two groups using SAS. SAS Global Forum 2012: Statistics and Data Analysis; Paper 335-2012.

46. Lin DY, Wei LJ, Ying Z. Checking the Cox model with cumulative sums of martingale-based residuals. Biometrika. 1993;80(3):557-572.

47. Lee AK, Warren B, Lee CJ, et al. The association of severe hypoglycemia with incident cardiovascular events and mortality in adults with type 2 diabetes. Diabetes Care. 2018;41(1):104-111.

48. Goto A, Goto M, Terauchi Y, Yamaguchi N, Noda M. Association between severe hypoglycemia and cardiovascular disease risk in Japanese patients with type 2 diabetes. J Am Heart Assoc. 2016;5(3):e002875.

49. Standl E, Stevens SR, Armstrong PW, et al. Increased risk of severe hypoglycemic events before and after cardiovascular outcomes in
TECOS suggests an at-risk type 2 diabetes frail patient phenotype Diabetes Care. 2018;41(3):596-603.

50. Mellbin LG, Rydén L, Riddle MC, et al. ORIGIN Trial Investigators. Does hypoglycaemia increase the risk of cardiovascular events? A report from the ORIGIN trial. Eur Heart J. 2013;34(40):3137-3144.

51. Zinman B, Marso SP, Christiansen E, et al. Hypoglycemia, cardiovascular outcomes, and death: The LEADER Experience. Diabetes Care. 2018;41(8):1783-1791.

52. Freemantle N, Danchin N, Calvi-Gries F, Vincent M, Home P. Relationship of glycaemic control and hypoglycaemic episodes to 4-year cardiovascular outcomes in people with type 2 diabetes starting insulin. Diabetes Obes Metab. 2016;18(2):152-158.

53. Monami M, Marchionni N, Mannucci E. Long-acting insulin analogues versus NPH human insulin in type 2 diabetes: a meta-analysis. Diabetes Res Clin Pract. 2008;81(2):184-189.

54. Horvath $\mathrm{K}$, Jeitler $\mathrm{K}$, Berghold $\mathrm{A}$, et al. Long-acting insulin analogues versus NPH insulin (human isophane insulin) for type 2 diabetes mellitus. Cochrane Database Syst Rev. 2007;(2):CD005613.

\section{SUPPORTING INFORMATION}

Additional supporting information may be found online in the Supporting Information section at the end of the article.

How to cite this article: Kuo S, Yang C-T, Wu J-S, Ou H-T. Effects on clinical outcomes of intensifying triple oral antidiabetic drug (OAD) therapy by initiating insulin versus enhancing OAD therapy in patients with type 2 diabetes: $A$ nationwide population-based, propensity-score-matched cohort study. Diabetes Obes Metab. 2019;21:312-320. https://doi.org/10. 1111/dom.13525 